\title{
Why Voluntary Postural Training Improves Recovery of Mental and Motor Functions in Patients with Traumatic Brain Injury?
}

\author{
L. Zhavoronkova ${ }^{1}$, A. Zharikova ${ }^{1,2}$, O. Maksakova ${ }^{2}$ \\ ${ }^{1}$ Institute of Higher Nervous Activity and Neurophysiology, Russian Academy of Sciences, Moscow, Russia \\ ${ }^{2}$ Burdenko Neurosurgery Institute, Department of Neurorehabilitation, Russian Academy of Medical Sciences, Moscow, Russia \\ Email: Lzhavoronkova@hotmail.com
}

Received July 24, 2013; revised August 29, 2013; accepted September 15, 2013

Copyright (C) 2013 L. Zhavoronkova et al. This is an open access article distributed under the Creative Commons Attribution License, which permits unrestricted use, distribution, and reproduction in any medium, provided the original work is properly cited.

\begin{abstract}
There are data about the positive effect of rehabilitation with postural training in traumatic brain injury (TBI) patients. Brain mechanisms of functional recovery have been investigated insufficiently. In this study, functional recovery was examined in TBI patients with primary motor (20 patients) or higher mental impairment (10 patients) - post-traumatic Korsakov syndrome compared to healthy volunteers $(\mathrm{n}=20)$. Integral clinical, stabilographic, and EEG approach was used as a tool for estimation of functional deficit in TBI patients before and after rehabilitation course. Specific alterations of stabilographic and EEG parameters were observed in TBI patients with primary motor or mental deficit before rehabilitation. Rehabilitation course with postural training improved recovery of all functions in both patient groups. To understand brain mechanisms of rehabilitation, we examined EEG findings during standing and postural task performance in healthy volunteers $(n=10)$. EEG coherence increase was marked for different spectral bands predominantly in the right hemisphere and more in alpha-2 and beta bands during postural task performance. These EEG findings were discussed in relation to rehabilitation effect on TBI patients and proved that postural training with visual feedback may provoke primary activation of the right hemisphere and can serve as a trigger for integration of cortical and subcortical structures and for recovery of motor and higher mental function in TBI patients.
\end{abstract}

Keywords: Traumatic Brain Injury; Rehabilitation; Postural Control; EEG Coherence

\section{Introduction}

Severe traumatic brain injury (TBI) is the most common cause for death and chronic disability in young people. Serious post-traumatic impairments arise due to the multicomponent nature of brain injuries in these patients. Consequences of TBI are often associated with impairment in different spheres-motor, cognitive, psychological, behavioral, emotional deficits and etc. Interaction derangement of various afferent and efferent systems (visual, vestibular, sensori-motor, and others) may be the main reason for people after TBI being unable to perform different simple and complex behaviors including maintenance of vertical posture $[1,2]$. On the other hand, clear link of mental, cognitive and memory deficits in TBI and brain damaged patients was ascertained $[3,4]$. Moreover, mild traumatic TBI known as concussion as a rule also was accompanied by long-term memory and cognitive deficit $[5,6]$. In any cases, consequences of severe TBI may pro- duce post-traumatic Korsakov syndrome (KS), which develops in the form of antero- and retrograde amnesia, disorientation in space and time, the emotional-personality sphere, etc. [7].

Recent advances in brain imaging methodologies have revealed important information regarding both structural and functional properties of the TBI patients [8-12]. Diffusion tensor imaging (DTI), which allows assessment of the severity of lesions to the white matter of the brain and conducting pathways of the brain, has been proposed as sensitive biomarker of TBI. A large number of researches using DTI studies in TBI patients compared with controls described reduction of fractional anisotropy of corticospinal tract, in the corpus callous and etc. [10-12].

However, traditional analysis of the brain-behavior correlation in healthy persons and in patients with different brain disorders is mostly implemented by focusing on alteration of brain signals - electroencephalogram (EEG) which is widely used in scientific researches and clinical 
practice [13]. Coherence analysis seems to be suitable for studying brain functions after TBI too, as it measures possible mechanisms of integration-disintegration). EEG coherence as a measure of spectral similarity may estimate cortical coupling between two signals as a function of frequency and reflect the structural connections and functional coupling between these regions [14-20].

Restoration of impaired functions in patients after severe TBI does require special rehabilitation techniques recruiting intact brain and body capacities and adaptivecompensatory potentials [21-24]. Memory and motor functions are known to be tightly interrelated as a rule. These deficiencies occur simultaneously in different types of brain pathology including TBI [25]. Neuropsychological approaches proposed by Luria and his followers are based on persisting functionality of impaired higher mental functions including memory and motor skills (especially professional) during rehabilitation [26]. Recent studies have shown that the inclusion of stabilotraining (ST) with feedback (voluntary postural training) into TBI rehabilitation programs is an effective operant approach promoting the recovery of postural skill [21-24]. Our previous data and results of other studies demonstrated restoration not only motor, but also memory functions due to ST as a special rehabilitation method [27,28]. This rehabilitation approach was based on Luria's hypothesis that motor memory was the most ancient type of memory. The motor (or postural) training may build up the basement for the recovery of more complex forms of behavior [22-25].

In previous studies, we used complex evaluation of patients' condition including quantitative clinical assessment scales, stabilographic and EEG coherence measures that were highly informative in following rehabilitation effects $[27,28]$. Now we'd like to seize neurophysiologic mechanisms of both motor and mental recovery of TBI patients with ST-based voluntary postural training. In view of the aforesaid, we set series of problem:

1) To compare ST and EEG features in primary motor deficit and primary mental impairment after severe TBI (Post-traumatic KS).

2) To analyse the peculiarities of restoration of motor and mental functions when voluntary postural training with ST is a considerable part of rehabilitation program.

3) To study main features of EEG reorganization in voluntary postural control in healthy volunteers. This part is available for understanding neurophysiologic mechanisms charged with rehabilitation effect of ST.

\section{Materials and Methods}

\subsection{Participants}

The researches were performed according to the declaration of Helsinki and they were approved by the Institu- tional Review Board from Burdenko Neurosurgery Institute RAMS.

Group 1 of TBI patients

Period of research with ST was 1 - 6 months after TBI. 20 patients were included (mean age $28 \pm 6.9$ years, right-handed) with loss of consciousness (4 - 10 points on the Glasgow Coma Scale - GSC) due to TBI, and coma lasting 4 - 40 days. According MRI data $65 \%$ of patients (13 of 20) had double-sided lesions without marked lateralization, while $25 \%$ (5 patients) had multiple lesions mainly of the right hemisphere, and $10 \%$ (two patients) had multiple lesions mainly of the left hemisphere. Diffuse axonal injury was identified in seven patients $(30 \%)$. Severe motor deficit in the form of inability for independent vertical maintenance was the main problem of all the group 1 patients.

Group 2 of TBI patients

A total of 10 patients with the sequelae of TBI and diagnoses of posttraumatic KS took part in the study; eight patients were aged $26-30$ (mean $26.14 \pm 3.18$, righthanders) years and two were aged over 50 years. Brain lesions were localised with $\mathrm{CT}$ and MRI. Loss of consciousness lasted 2 - 40 days with GSC 5 - 8 points.

Group 3 of participants

Twenty healthy subjects' data (mean age $26 \pm 3.5$ years, right-handers) served as controls for stabilographic studies, and EEG investigations (sitting at the chair with closed eyes). Ten healthy volunteers were studied with telemetric EEG (sitting, standing, and performing voluntary postural control task with biofeedback).

\subsection{Methods and Procedures}

\subsubsection{Clinical Study}

Neurologic examination, stabilographic, and EEG investigations were performed in all patients before and after rehabilitation courses including stabilotraining with biofeedback (RC-ST). Integral quantitative assessment of patients' functional state was appraised with the MayoPortland Adaptability Inventory 3 (MPAI), which provides a differential analysis of deficits in motor and cognitive (including memory) functions, as well as emotional status and the level of social adaptation (maximal deficit - four points, normal score is 0) [29].

\subsubsection{Stabilographic Study}

Stabilographic study was assessed using MBN-Biomekhanika system (MBN, Russia), consisting of a special force platform connected with computer system. RC-ST courses consisted of 8 - 15 stabilotraining sessions (30 $40 \mathrm{~min}$ ) over a period of 4 - 6 weeks. Every session was a set of static and dynamic motor tasks of various difficulties. Each session started and ended by balance maintenance on the force platform with open and closed eyes 
without any tasks. Set of stabilographic parameters obtained in this condition was used to analyze the rate (V) of oscillations in center of pressure (COP) and amplitude of COP oscillations in the frontal (Af) and sagittal planes (As). The simplest task was the "identical bars" one. To perform this task, volunteers and patients had to keep equal heights of two bars on a monitor screen (generated vs. platform signal). Coincidence of two bars positions with initial one was indicative for proper pose maintenance. More complex task ("different bars") corresponded to control marker movement upwards or downwards in random order with different amplitude. To perform the task (keep the bars lined up) volunteers and patients had to shift of COP to right or left leg. Series of complicated tasks embraced so called "shooter" test with the control marker moving along eight directions from monitor center. This task required rapid displacements COP to right, left, forwards, backwards, and diagonally. The training effects of RC-ST were evaluated in terms of a set of stabilographic parameters (V, Af and As) and task performance ( $\%$ success).

\subsubsection{EEG Recording and Processing}

Subjects were sitting in an arm-chair with eyes closed. EEG was recorded at rest state using of Nihon Cohden equipment (Japan). The electrical activity from the scalp was recorded at 19-cites: Fp1, Fz, Fp2, F3, F4, F7, F8, C3, Cz, C4, P3, Pz, P4, O1, O2, T3, T4, T5, T6 according to the International 10 - 20 system. The ground electrode was located to $\mathrm{Fz}$ with electrode impedances lower 5. Each EEG trace lasted at least 60 - $70 \mathrm{~s}$ and was recorded using a sampling frequency of $100 \mathrm{~Hz}$. The filters were set to $35 \mathrm{~Hz}$ with bandwidth of $0.5-30 \mathrm{~Hz}$. Artifact-free segments of monopolar EEG recordings with indifferent ear electrodes were used for further analysis. The segments containing eye blinks and artifacts were rejected. At last 50 - 60 s EEG segmented in epochs of 5c (10 epochs) were subjected for Fast Fourier Transformation and EEG coherence calculating. Band limits were defined as: delta $-0.5-3.9 \mathrm{~Hz}$; theta - 4.3 - 7.8; alpha8.2 - 12.9; beta - 13.324.3. The same EEG equipment was used for all the patients and for 20 healthy volunteers.

Telemetric EEG recording was carried out with Medicor hardware-and-software complex (Taganrog, Russia). EEG was recorded $\mathrm{Ag} / \mathrm{AgCl}$ electrodes mounted in 19 Electro-cap. The electrical activity from the scalp was recorded at the same 19-cites according to the International 10 - 20 system. EEG was recorded in healthy persons sitting and standing on the floor, on a force platform (in all tasks with the eyes open and closed), and during ST test, 60 - $70 \mathrm{~s}$ for each experimental situation. EEG was recorded using a sampling frequency of $200 \mathrm{~Hz}$ and in $\mathrm{DC}$ to $70-\mathrm{Hz}$ frequency range. The segments contain- ing eye blinks and artifacts were rejected. Last 45 - $50 \mathrm{~s}$ EEG's were segmented in epochs of $5 \mathrm{~s}$ (a total no less 10 epochs were taken). EEG power and coherence were calculated for following spectral bands: delta-2 - 3.9 Hz; theta - 4.39 - 7.8; alpha1-8.2 - 10.2; alpha2-10.5 12.9; beta-13.3 - 30.1; gamma-30.5 - 40.2.

\subsection{Statistic}

Stabilographic and clinical data were analyzed statistically using nonparametric methods on Statistica 6.1. The Mann-Whitney test was used to compare for independent groups, i.e., healthy subjects and patients, and the Wilcoxon test was used for linked sets (patients before and after ST). EEG data of both EEG recording systems were analyzed statistically using a program written by the authors [4] to determine the significance of differences (Mann-Whitney test) in the set of EEG coherence parameters by the paired comparisons method.

\section{Results}

\subsection{Clinical, Stabilographic and EEG Analysis in TBI Patients before and after ST}

\subsubsection{Clinical-Rehabilitation Estimation}

Deficits in all domains (motor, cognitive, and emotional) were revealed in patients of group 1 (Figure 1(a)) and group 2 (Figure 1(b)) by means of integral MPAI scale before rehabilitation courses, along with decreasing potential for social adaptation. According to MPAI movement sphere suffered the most in Group 1, whereas Group 2 (patients with KS) demonstrated strongly pronounced cognitive deficit. All patients improved their integral and differential rating after courses of rehabilitation with ST. Both motor and cognitive deficits reduced, along with a tendency to emotional improvement and social adaptation progress.

\subsubsection{Stabilographic Studies}

Results of stabilographic studies before RC-ST showed up the common changes in all patients of both groups during the maintenance of balance with eyes open and the eyes closed: increase of trajectory length (amplitude) of COP oscillations at the frontal and sagittal planes and higher rate of COP oscillations in comparing with healthy probationers (Figure 2). Rate oscillations were much higher in patients of Group 1 in compare with Group 2. One may consider this finding as evidence of greater efforts to balance maintenance in Group 1. Stabilographic parameters improved after RC-ST in all patients, it was indicative for regress of motor deficit as a whole. However none of the values reached the normal level. The most evident change was a decrease of COP oscillation rate, thereby indicating in energy charge reduction of balance maintaining. 
(a) 1st group of patients

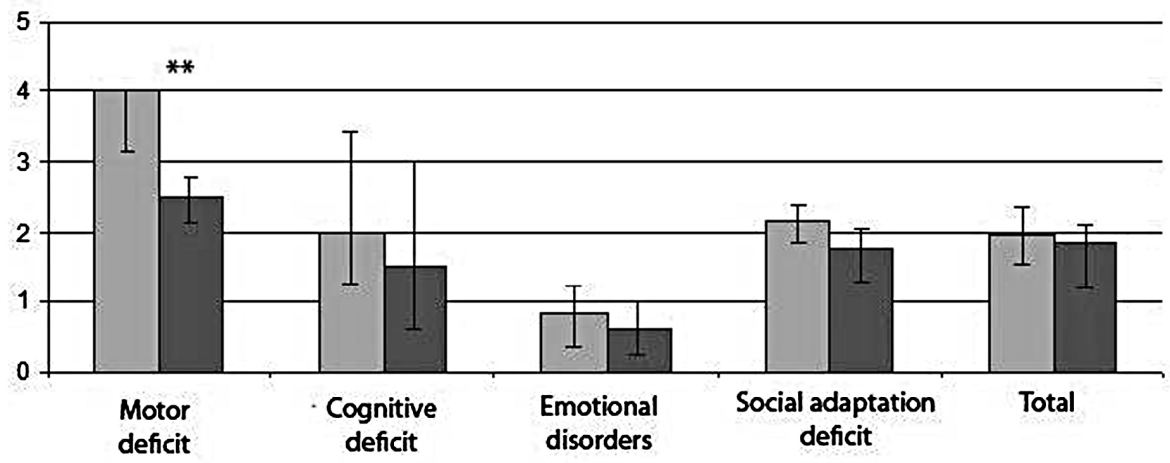

(b) 2nd group of patients

Before ST

After ST

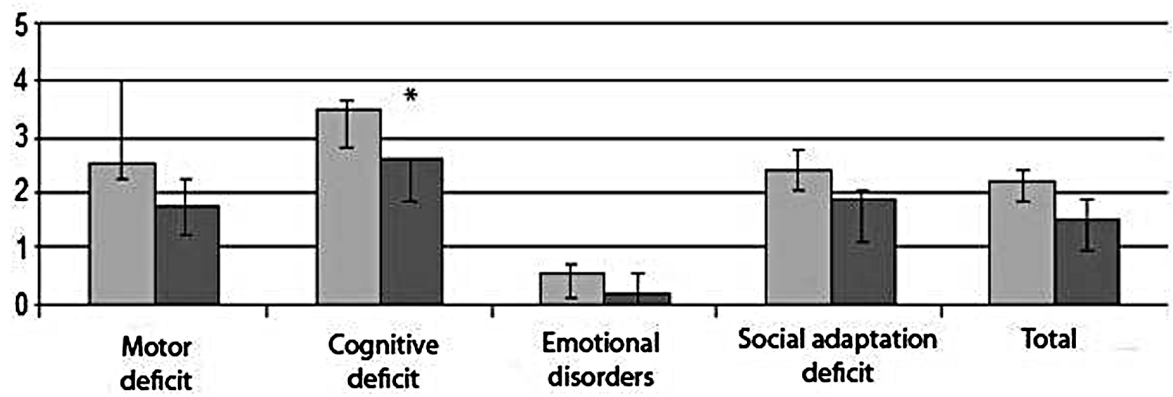

Figure 1. Comparison of differential and integral clinical assessments $(\mathrm{MPAI})$ in TBI patients of the $1^{\text {st }}(\mathrm{n}=20)$ and $2^{\text {nd }}(\mathrm{n}=$ 10) groups before and after courses of rehabilitation. Ordinate—severity of deficit, points.

(a) 1st group of patlents
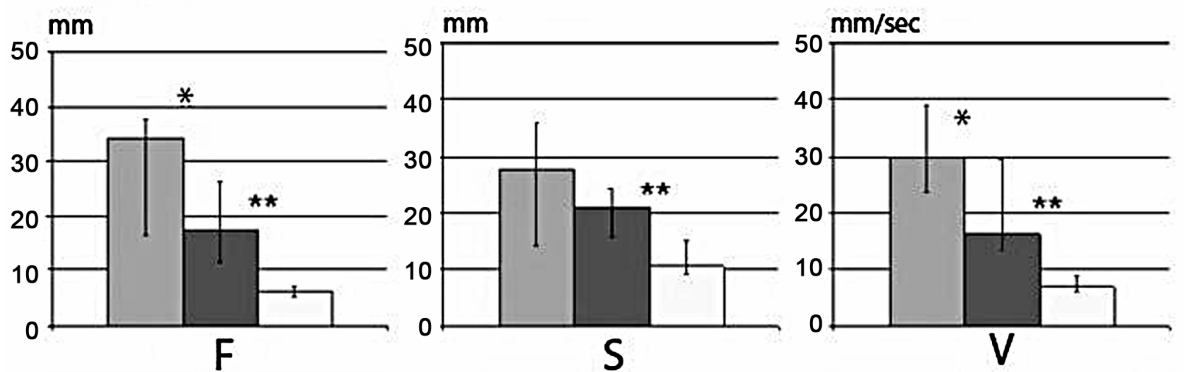

(b) 2 nd group of patients


Figure 2. Changes of stabilographic parameters in $1^{\text {st }}(n=20)$ and $2^{\text {nd }}(n=10)$ TBI patients before and after courses of rehabilitation in compare with healthy subjects $(n=20)$. The ordinate shows displacement of the center of pressure: AFamplitude of OCP oscillations at the frontal plane, mm; AS-amplitude of oscillatios at the sagittal plane, mm; V-rate of displacement, $\mathrm{mm} / \mathrm{sec}$. 
Stabilographic tasks' performance was worse in both groups before RC-ST, than in healthy volunteers (Figures 3(a) and (b)). It should be noted that at the initial stages of rehabilitation patients of Group 1 recognized instructions of tasks, but physically handicapped to execute, while patients of Group 2 (KS) experienced difficulties in understanding the tasks. So during the first 2 3 sessions $30 \%$ of patients with KS were unable to remember the exercise, and needed to be explaining each task immediately before start. After $4-6$ sessions these patients started to perform tasks without preliminary instructions just after observing the screen. It should be noted that during the first sessions KS patients performed task "Shot" slightly worse. One may consider it as difficulties of transfer from real body displacement in threedimensional space to screen presented two-dimensional space. After RC-ST quality of all tasks performance improved, especially task "Shot" in KS patients, while nobody ran up to normal quality.

\subsubsection{EEG Studies}

EEG coherence analysis in patients of Group 1 before RC-ST compared with normal data revealed predominant decreases of interhemispheric pairs for majority of cortical areas, maximal in the central and parietal areas, particularly for the alpha range (Figure 4(a)). This was accompanied by coherence increase of slow EEG rhythms. Parallel with re-learning of independent balance maintenance and ability to perform special tasks after RC-ST, EEG coherence has increased in comparison with preRC-ST levels. Maximal increase was marked in interhemispheric leads, particularly in central and parietal brain areas (Figure 4(b)). Along with this observation, there was coherence increase of alpha range for pairs of leads including the intrahemispheric pairs.

Comparison of EEG coherences in patients of Group 2 before RC showed maximal reductions for all frequency ranges, particularly alpha in frontal lobes (interhemispheric leads, especially) in compare with healthy subjects. Similar low coherences were in occipital-parietal hemispheric areas, greater extent on the right. In particular, there was a significant decrease of EEG coherence of alpha range for long diagonal pairs, i.e., between left frontal and right occipital-parietal areas. After RC-ST EEG coherence parameters in patients obviously changed: intra-hemispheric coherence increased, the most in right hemisphere with

\section{(a) 1st group of patients}
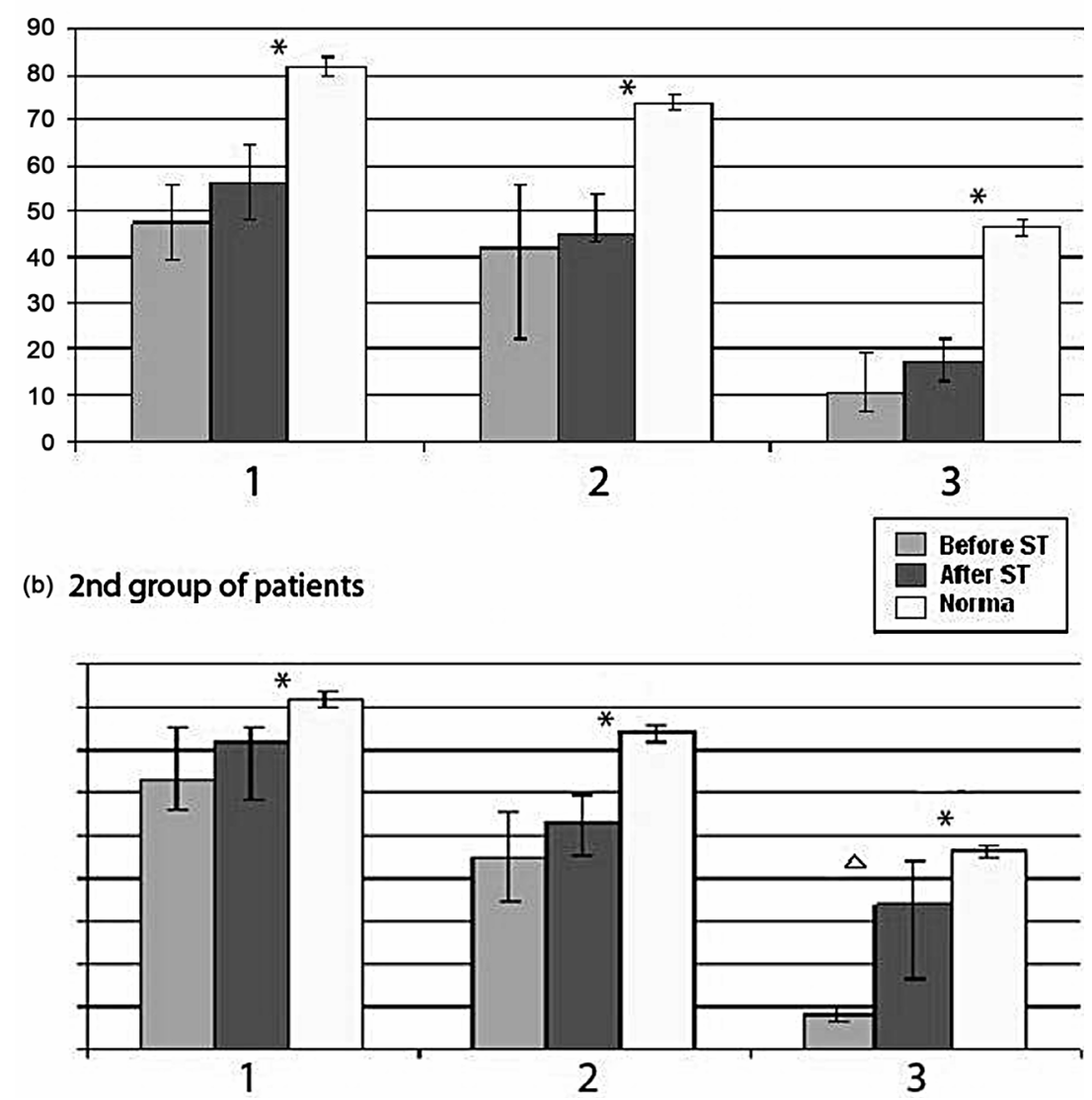

Figure 3. Successfulness in performing postural tasks before and after rehabilitation in TBI patients of Group 1 and Group 2 as compared with healthy subjects. The ordinate shows the success rate of task performance (\%). 


\section{Before rehabilitation}
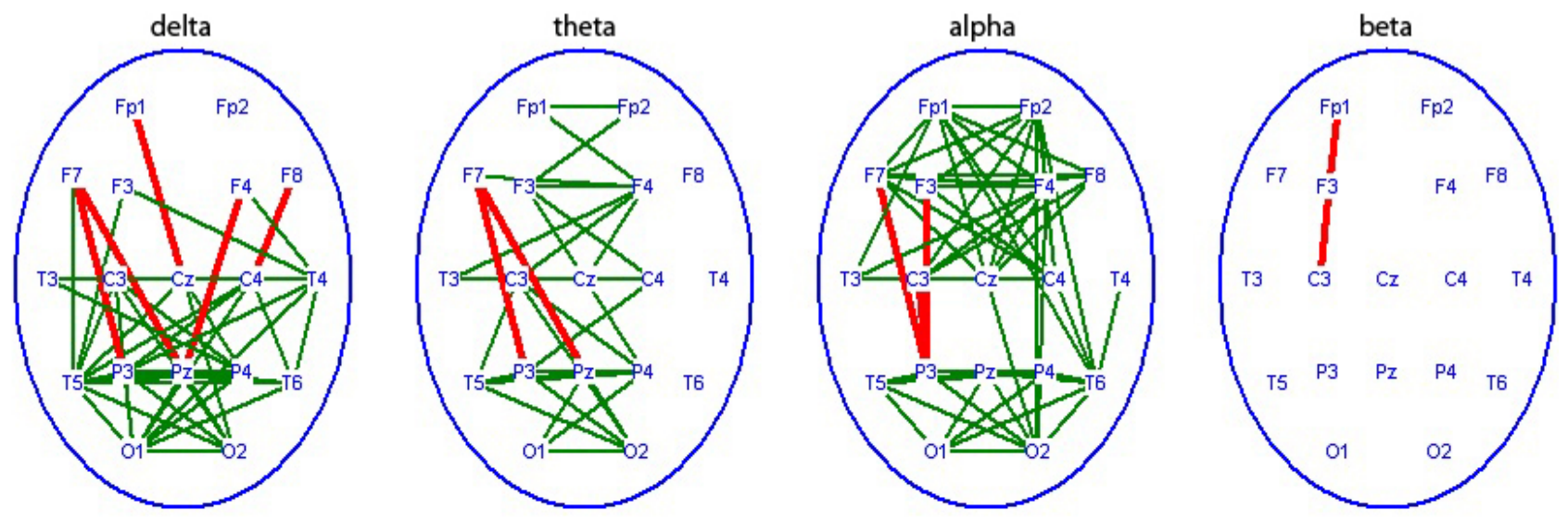

\section{After rehabilitation}
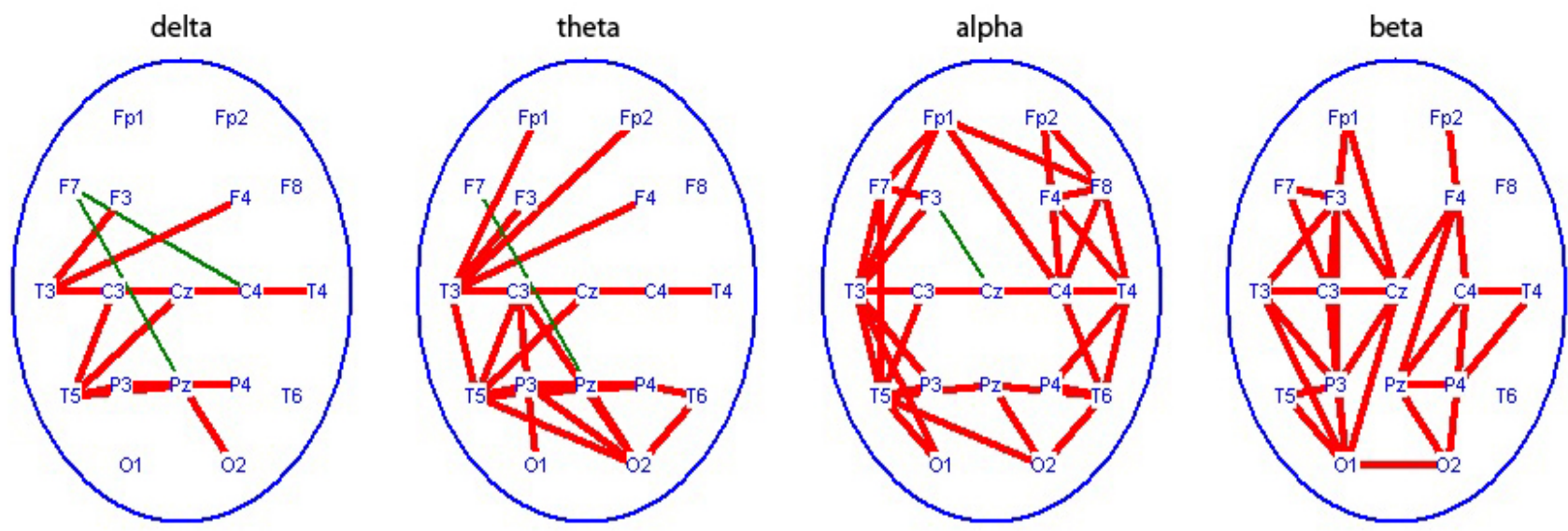

- Increase of coherence
— Decrease of coherence

Figure 4. Regional characteristics of coherence changes $(p<0.01)$ for different EEG rhythms in Group 1 (n = 20$)$ before RC-ST as compared with healthy subjects $(n=20)$ - top row and coherence changes in this patients after RC-SP as compared with pre-RC-ST level-bottom row.

values higher than normal, while inter-hemispheric coherence remained low (Figure 5(b)). EEG coherence tended to normalizing after ST, yet it differed from normal values in toto, possibly due to incomplete adaptation of these patients even after rehabilitation course with ST.

\subsection{EEG-Markers Upright Vertical Position in Healthy Persons}

Results of EEG study showed, that if healthy volunteers with their eyes open passed from sitting to standing position EEG coherence increased in beta-range, mainly in central regions, then it may decrease in other cortex regions (Figure 6). If this transition was performed with closed eyes EEG coherence had increased practically in all the rhythm ranges especially in right hemisphere, mostly for theta, alpha and beta-bands. Let's note marked coherence increase observed in the fronto-central regions. It may indicate additional executive functions' control in absence of visual correction. As far as the task complicated when a subject closed eyes standing on the force platform, EEG coherence clear increased in delta, theta, and alpha bands, especially in frontal and central regions revealing additional efforts of balance maintenance. Coherence values increased particularly in the right hemisphere for different EEG rhythms. ST tasks performance (in compare with simple standing on force platform) was accompanied by EEG coherence increase mainly in right hemisphere, the greatest for alpha and beta bands, and for beta band in frontal areas. 


\section{Before rehabilitation}
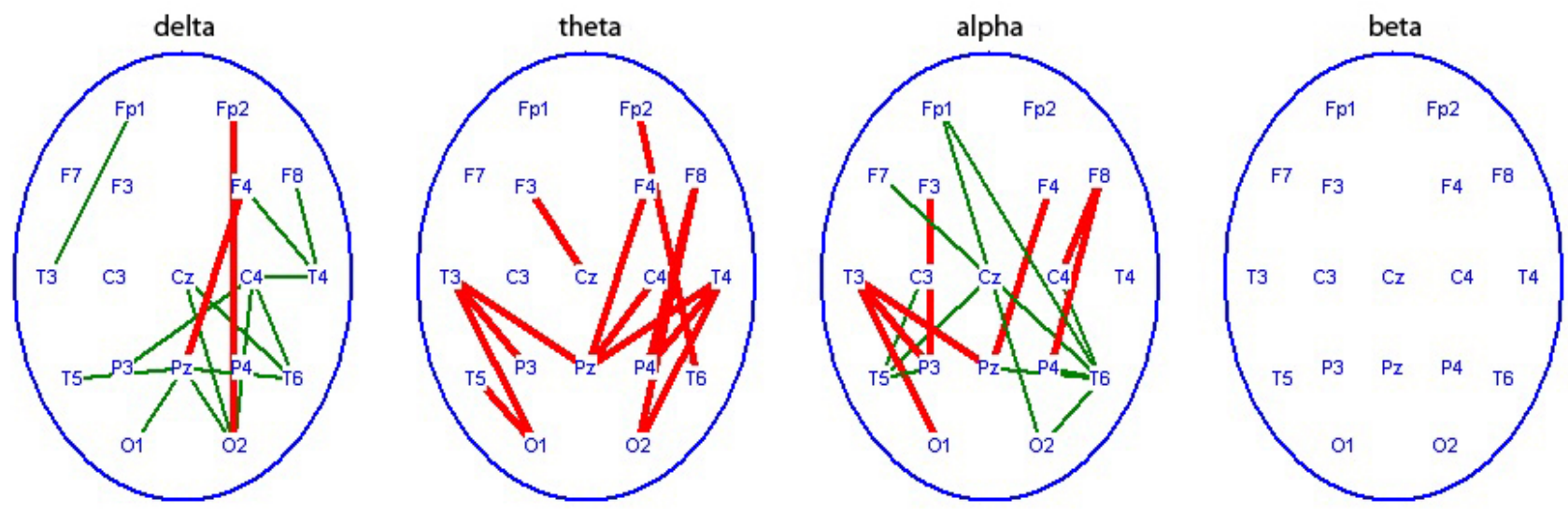

\section{After rehabilitation}
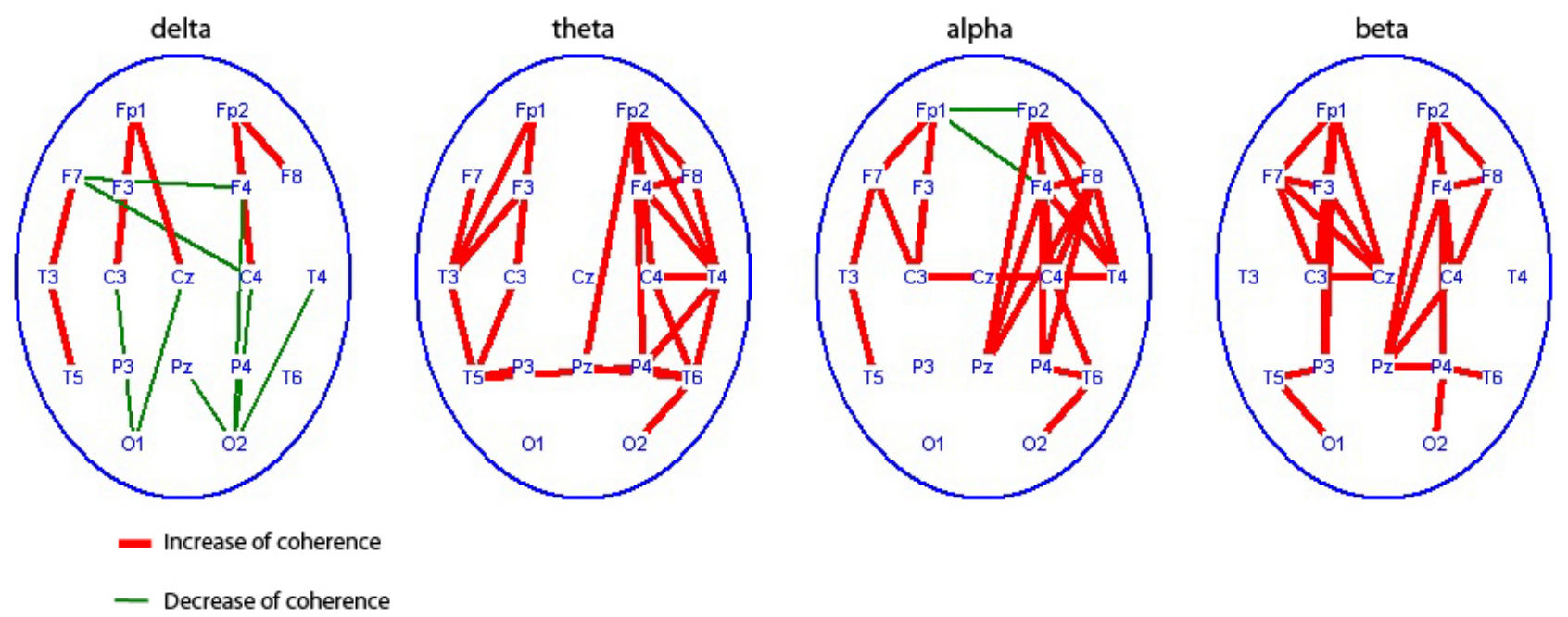

Figure 5. Regional characteristics of significant coherence changes $(p<0.0)$ of different EEG rhythms in patients with TBI of the 2nd group $(n=10)$ before courses rehabilitation as compared with healthy subjects $(n=20)$. - top row and coherence changes in this patients after RC-SP as compared with pre- RC-ST level-bottom row.

\section{Discussion}

Jointly using of clinical-rehabilitation scales, stabilographic and EEG analysis before rehabilitation course current study has revealed some specific impairment in TBI patients with predominant motor or higher mental deficits. The rate of CPO fluctuations (stabilography) was higher in patients with primary motor deficit, thus reflecting their harder costs to maintain balance in compare with KS patients. These results are supported by previous findings of increased CPO values in patients surviving brain damage, and minimal CPO values in professional athletes [30,31]. On the other hand, stabilographic tasks requiring body displacement in three-dimensional space presented maximal problems for KS patients with minimal movement deficit. So, analysis of performance qual- ity of stabilographic tasks experimentally confirmed space perception disorders found out in KS by previous clinic studies [7].

Our EEG data showed the lower coherence values in TBI patients than normal as reflection of coupling suffer especially between distant cortical areas in both patients' groups before rehabilitation. There are some EEG findings related to damage cortical connectivity as a result of sport injury [32]. On the other hand, DTI data being a promising tool to detect axonal injury demonstrated a primary damage of long cortical-cortical and corticalsubcortical axonal ways especially of corpus callosum in TBI patients [8-10]. These data confirm the idea of conduction pathways damage being one of major consequences of brain injury that goes with disintegration (uncoupling) of functionally significant brain regions, and 


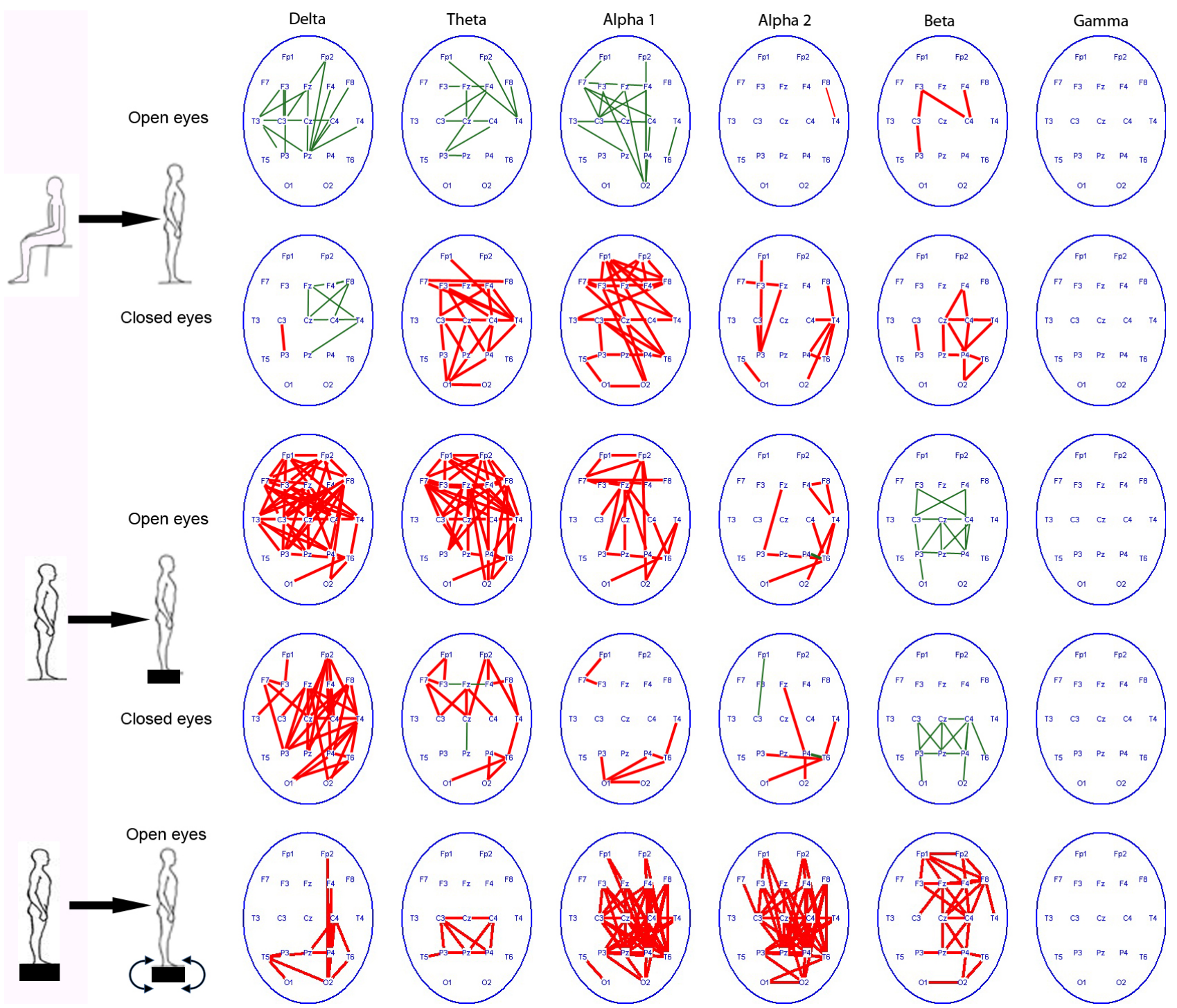

Figure 6. Changes of EEG coherence $(p<0.01$, decrease - green lines or increase - red lines) in healthy volunteers $(n=10)$ after transition from a sitting position to standing on the floor, transition to standing on the floor to the force platform with open and closed eyes; the standing position on the force platform at the rest state to the performing of stabilographic task with the eyes open.

after all with disabilities [8-10].

Furthermore we described peculiarities of EEG coherence changes in groups of patients with predominant motor or higher mental deficits. The lowest EEG coherence values were at the central and parietal cortical areas (zones of motor and postural presentation); it was a specific feature of patients with mainly motor deficit. While in KS patients EEG coherence was minimal between left frontal and right occipital-parietal cortical areas along so-called "cognitive axis", going with disability to make appropriate spatial assessments, to switch attention from one type of activity to another, and to place events and actions on the time scale $[27,28]$.

A comparison of the integrated parameters in TBI patients before and after courses of rehabilitation including voluntary postural training with visual feedback demonstrates recovery of motor (postural) as well as mental functions after rehabilitation. Some explorers found the similar results in brain-injured patients during rehabilitation course with ST [21-23,30,31]. At the same time, there is a problem of the direct role of ST as compared with other rehabilitation procedures after TBI and also with spontaneous recovery processes that can proceed for a long time after trauma. However some previous research showed that rehabilitation with ST gave a higher gain in recovery of patients with different form of brain pathology than programs without ST [24].

We proposed that positive effect of rehabilitation with postural training supports Luria's hypothesis as motor training might serve a trigger of recovery both motor as 
well as higher mental functions [28]. Yet a patient faced immediately with results of postural task performance due to visual feedback attracting his attention and switching on his motivation.

It seems important that EEG coherence increased maximally after RC-ST in interhemispheric pairs with the highest values in central and parietal areas for different spectral bands. Taking into account genesis of EEG rhythms these data may reflect functional integration between different cortical and subcortical structures for provision of the postural control during this rehabilitation approach $[33,34]$. Data obtained in recent years using DTI directly confirm this EEG finding. So, EEG and DTI data allow supposing that functional integration emerges both due to new pathways' formation and to recruitment of previously unused surviving structures and connections as a result of specific rehabilitation arrangement.

New data of mammals' experiment have appeared recent years supporting the important role of motor training in neurogenesis. Particularly it should be noted the following: animal experiments have shown this process is more apparent in individuals with active seeking behavior combined with elevated motor activity [35]. Recent neuroimaging studies in TBI patients demonstrated fundamental potentialities of new axonal pathways forming associated with successful rehabilitation outcome [11]. On the whole the results of these studies are optimistic in terms of new neurorehabilitation approaches for patients surviving severe brain injury.

Successful outcome of rehabilitation with postural training may be explained by an important biological role in supporting vital functions during the formation of a human's ability to stand upright during evolution. Comparison of EEG coherence increase for different spectral bands in healthy persons when standing up and performing of postural tasks with data of the EEG rhythm generation support the concept of dynamic interaction between the hierarchically organized brain structures, both cortical and subcortical $[34,35]$. In regard to our data EEG coherence was increased predominantly in right hemisphere in standing and postural task performance. It is known that morpho-functional properties of human brain are asymmetric with larger volume of white matter and density of axonal fibers in right hemisphere [36]. Besides, clinical and EEG studies showed the tighter functional connections of the right hemisphere with the vitally important diencephalon structures than of the left one. Numerous neurorehabilitation approaches are based on procedures promoting primary activation of right hemisphere: emotionally significant stimuli, music therapy, aromatherapy, etc. $[37,38]$. It may to mean particular important role of right hemisphere activation in recovery process after TBI.

At last, increase of EEG coherence at the frontal cor- tical areas during performance of voluntary postural tasks support the notion of the dynamic interaction between the hierarchically organized brain structures with the leading role of the brain's cortex in executive functions [39].

\section{Conclusion}

Integral clinical, stabilographic, and EEG approach is an informative tool for estimation of different functional disorders in TBI patients before and after rehabilitation course. Specific alterations of stabilographic and EEG parameters may be markers of primary motor or higher mental deficit observed in different terms after TBI. EEG parameters may provide evidence of activating cortical and subcortical integration (interaction), mainly in right hemisphere during standing position and performance of postural tasks. We propose that rehabilitation approach including voluntary postural training with visual feedback serves as a trigger and an integrator in the recovery of impaired motor and higher mental function in TBI patients.

\section{REFERENCES}

[1] A. Maas, N. Stocchetti and R. Bullock, "Moderate and Severe Traumatic Brain Injury in Adults," Lancet Neurology, Vol. 7, No. 8, 2008, pp. 728-741. http://dx.doi.org/10.1016/S1474-4422(08)70164-9

[2] A. Helmy, M. Vizcaychipi and A. K. Gupta, "Traumatic Brain Injury: Intensive Care Management," British Journal of Anaesthesia, Vol. 99, No. 1, 2007, pp. 32-42.

[3] B. Levine and E. Fujiwara, "In Vivo Characterization of Traumatic Brain Injury Neuropathology with Structural and Functional Neuroimaging," Journal of Neurotrauma, Vol. 23, No. 10, 2006, pp. 1396-1411.

[4] D. Plummer, P. Amato, D. Saracino and E. Fox, "Interactions between Cognitive Tasks and Gait after Stroke: A Dual Task Study," Gait and Posture, Vol. 27, No. 4, 2008, pp. 683-6801.

[5] S. Slobounov, M. Gay and B. Iohnson, "Concussion in Athletes: Ongoing Clinical and Brain Imaging Research Controversies," Brain imaging and Behavior, Vo. 6, No. 2, 2012, pp. 224-243. http://dx.doi.org/10.1007/s11682-012-9167-2

[6] A. Maas, N. Stocchetti and R. Bullock, "Moderate and Severe Traumatic Brain Injury in Adults," Lancet Neurology, Vol. 7, No. 8, 2008, pp. 728-741. http://dx.doi.org/10.1016/S1474-4422(08)70164-9

[7] H. Oyama, T. Mabuchi, M. Niwa, Y. Kida, T. Tanaka, K. Yoshida, T. Iwakoshi, R. Kitamura, S. Maezawa and T. Kobayashi, "Traumatic Korsakoff Syndrome," Journal of Clinical Neuroscience, Vol. 5, No. 4, 1998, pp. 441-444. http://dx.doi.org/10.1016/S0967-5868(98)90284-3

[8] E. D. Bigler and E. Wilde E., "Quantitative Neuroimaging and the Prediction of Rehabilitation Outcome Following Traumatic Brain Injury," Frontiers in Human Neuroscience, Vol. 4, 2010, pp. 228-230. 
[9] P. E. Vos and E. D. Bigler, "White Matter in Traumatic Brain Injury: Dis- or Disconnection?" Neurology, Vol. 77, No. 9, 2011, pp. 810-811.

[10] A. Sidaros, A. Engberg, K. Sidaros, M. G. Liptrot, M. Herning, P. Petersen, O. B. Paulson, T. L. Jernigan and E. Rostrup, "Diffusion Tensor Imaging during Recovery from Severe Traumatic Brain Injury and Relation to Clinical Outcome: A Longitudinal Study," Brain, Vol. 131, No. 2, 2008, pp. 559-572.

http://dx.doi.org/10.1093/brain/awm294

[11] A. Sidaros, A. Skimminge, M. G. Liptrot, K. Sidaros, A. W. Engberg, M. Herning, O. B. Paulson, T. L. Jernigan and E. Rostrup, "Long-Term Global and Regional Brain Volume Changes Following Severe Traumatic Brain Injury: A Longitudinal Study with Clinical Correlates," NeuroImage, Vol. 44, No. 1, 2008, pp. 1-8.

[12] R. Hurley, A. Hayman and K. Taber, "Traumatic Axonal Injury: Novel Insights into Evolution and Identification," Journal of Neuropsychiatry \& Clinical Neurosciences, Vol. 16, No. 1, 2004, pp. 125-129.

[13] E. Niedermeyer and F. Lopes da Silva, "Electroencephalography: Basic Principles, Clinical Applications and Related Fields," 5th Edition, Lippinkot Williams \& Wilkins, Philadelphia, 2005.

[14] J. C. Shaw, K. P. O'Connor and O. C. Ongley, "EEG Coherence as a Measure of Cerebral Functional Organization. Architectonics of Cerebral Cortex," N.Y. Raven Press, New York, 1978.

[15] R. W. Thatcher, P. J. Krause and M. Hrybyk, "CorticoCortical Associations and EEG Coherence: A Two-Compartmental Model," Electroencephalography and Clinical Neurophysiology, Vol. 64, No. 2, 1986, pp. 123-143.

[16] V. S. Rusinov, O. M. Grindel, G. N. Boldyreva and E. M. Vakar, "Human Brain Biopotentials (Mathematical Analysis)," Medicine, Moscow, 1987.

[17] M. N. Livanov, "Spatial-Temporal Organization of Potential and System Activity of the Brain," Sciences, Moscow, 1989.

[18] P. Rappelsberger and H. Petsche, "Probability Mapping: Power and Coherence Analysis of Cognitive Processes," Brain Topography, Vol.1, No. 1, 1998, pp. 46-54.

[19] D. Ruchkin, "EEG Coherence," International Journal of Psychophysiology, Vol. 57, No. 2, 2005, pp. 83-85.

[20] D. B. Chlorich, A. E. Rangaswamy and B. Porjesz, "EEG Coherence: Topography and Frequency Structures," Experimental Brain Research, Vol. 198, No. 1, 2009, pp. 59-83. http://dx.doi.org/10.1007/s00221-009-1936-9

[21] F. Eser, G. Yavuzer, D. Karakus and B. Karaoglan, "The Effect of Balance Training on Motor Recovery and Ambulation after Stroke: A Randomized Controlled Trial," European Journal of Physical and Rehabilitation Medicine, Vol. 44, No. 1, 2008, pp. 19-25.

[22] F. Benvenuti, R. Mecacci and I. Gineprari, "Kinematic Characteristics of Standing Disequilibrium: Reliability and Validity of a Posturographic Control," Archives of Physical Medicine and Rehabilitation, Vol. 80, No. 3, 1999, pp. 278-287. http://dx.doi.org/10.1016/S0003-9993(99)90138-7
[23] M. de Haart, A. Geurts, S. C. Huidekoper, L. Fasotti and J. Limbeek, "Recovery of Standing Balance in Postacute Patients: A Rehabilitation Cohort Study," Archives of Physical Medicine and Rehabilitation, Vol. 85, No. 6, 2004, pp. 886-894.

http://dx.doi.org/10.1016/j.apmr.2003.05.012

[24] L. Zhavoronkova, O. Maksakova and G. Schekutiev, "Effect of Stabilotraining on Postural Control Recovery at Different Stages of Rehabilitation after Sever Traumatic Brain Injury," From Basic Motor Control to Functional Recovery, Sofia, 2007.

[25] N. D. Schiff, "Recovery of Consciousness after Brain Trauma: Mesocircuit Hypothesis," Trend in Neuroscience, Vol. 204, No. 1, 2010, pp. 1-9.

[26] A. R. Luria, "Stage of the Path," Moscow State University Press, Moscow, 2001.

[27] L. A. Zhavoronkova, O. A. Maksakova, A. V. Zharikova, I. S. Flerov, G. A. Schekutiev and V. L. Naidin, "Effect Including Stabilotraining in Rehabilitation of Patients with Post-Traumatic Korsakov Syndrome," Neuroscience and Behavioral Physiology, Vol. 42, No. 4, 2012, pp. 484490.

[28] L. A. Zhavoronkova, A. V. Zharikova and O. A. Maksakova, "The Integrating Role of Restoration of Voluntary Postural Control in Rehabilitation of Patients with Craniocerebral Trauma," Neuroscience and Behavioral Physiology, Vol. 42, No. 5, 2012, pp. 486-494.

[29] J. F. Malec, "Comparability of Mayo-Portland Adaptability Inventory Ratings by Staff, Significant Others and People with Acquired Brain Injury," Brain Injury, Vol. 18, No. 6, 2004, pp. 563-575.

[30] A. C. Gerts, G. M. Ribbets, J. A. Knoop and J. Limbeek, "Identification of Status and Dynamic Postural Instability Following Traumatic Brain Injury," Archives of Physical Medicine and Rehabilitation, Vol. 6, No. 4, 1996, pp. 639- 644.

[31] R. Balasubramaniam and A. Wing, "The Dynamics of Standing Balance," Trends in Cognitive Sciences, Vol. 6, No. 12, 2002, pp. 112-125.

[32] C. Cao and S. Slobounov, "Alteration of Cortical Functional Connectivity as Result of Traumatic Brain Injury Revealed by Graph Theory, ICA, and sLORETA Analysis of EEG Signals," IEEE Transactions on Neural Systems and Rehabilitation, Vol. 18, No. 1, 2010, pp. 11-19.

[33] F. N. Lopez da Silva, "Neural Mechanisms Underlying Brain Waves: From Neural Membrane to Networks," Electroencephalography and Clinical Neurophysiology, Vol. 79. No. 2, 1991, pp. 81-90.

[34] G. Buzsaki, "Rhythms of the Brain," Oxford University Press, New York, 2006. http://dx.doi.org/10.1093/acprof:oso/9780195301069.001. 0001

[35] X. Gao, G. Enikolopov and J. Chen, "Direct Isolation of Stem Cells in Adult Hippocampus after Traumatic Injury," Journal Neurotrauma, Vol. 25, No. 8, 2008, pp. 985-995. http://dx.doi.org/10.1089/neu.2008.0460

[36] S. F. Witelson, "Brain Asymmetry, Functional Aspects," In: States of Brain and Mind, Birhauser, Boston \& Basel, 
1988.

[37] N. Bragina and T. Dobrokhotova, "Human Functional Asymmetry," Medicine, Moscow, 1981.

[38] E. Goldberg and L. Costa, "Hemispheric Differences in Aquisition and Use of Discriptive System," Brain and
Language, Vol. 14, No. 2, 1981, pp. 144-173.

[39] C. Maurer, R. Mergner and R. Peterka, "Multisensory Control of Human Upright Stance," Experimental Brain Research, Vol. 171, No. 3, 2006, pp. 231-250. 